\title{
Identification of Yersinia enterocolitica isolates from humans, pigs and wild boars by MALDI TOF MS
}

Katarzyna Morka', Jarosław Bystroń², Jacek Bania², Agnieszka Korzeniowska-Kowal' , Kamila Korzekwa', Katarzyna Guz-Regner ${ }^{1}$ and Gabriela Bugla-Płoskońska ${ }^{1 *}$ (D)

\begin{abstract}
Background: Yersinia enterocolitica is widespread within the humans, pigs and wild boars. The low isolation rate of $Y$. enterocolitica from food or environmental and clinical samples may be caused by limited sensitivity of culture methods. The main goal of present study was identification of presumptive $Y$. enterocolitica isolates using MALDI TOF MS. The identification of isolates may be difficult due to variability of bacterial strains in terms of biochemical characteristics. This work emphasizes the necessity of use of multiple methods for zoonotic $Y$. enterocolitica identification.

Results: Identification of $Y$. enterocolitica isolates was based on MALDI TOF MS, and verified by VITEK 2 Compact and PCR. There were no discrepancies in identification of all human' and pig' isolates using MALDI TOF MS and VITEK 2 Compact. However three isolates from wild boars were not decisively confirmed as Y. enterocolitica. MALDI TOF MS has identified the wild boar' isolates designated as $3 \mathrm{dz}, 4 \mathrm{dz}, 8 \mathrm{dz}$ as $Y$. enterocolitica with a high score of matching with the reference spectra of MALDI Biotyper. In turn, VITEK 2 Compact identified $3 \mathrm{dz}$ and $8 \mathrm{dz}$ as Y. kristensenii, and isolate $4 \mathrm{dz}$ as $Y$. enterocolitica. The PCR for $Y$. enterocolitica 165 rDNA for these three isolates was negative, but the $16 S$ rDNA sequence analysis identified these isolates as Y. kristensenii ( $3 \mathrm{dz}, 4 \mathrm{dz})$ and $Y$. pekkanenii $(8 \mathrm{dz})$. The wild boar' isolates $3 \mathrm{dz}, 4 \mathrm{dz}$ and $8 \mathrm{dz}$ could not be classified using biotyping. The main bioserotype present within pigs and human faeces was 4/O:3. It has been shown that $Y$. enterocolitica 1B/O:8 can be isolated from human faeces using ITC/CIN culturing.

Conclusion: The results of our study indicate wild boars as a reservoir of new and atypical strains of Yersinia, for which protein and biochemical profiles are not included in the MALDI Biotyper or VITEK 2 Compact databases. Pigs in the south-west Poland are the reservoir for pathogenic Y. enterocolitica strains. Four biochemical features included in VITEK 2 Compact known to be common with Wauters scheme were shown to produce incompatible results, thus VITEK 2 Compact cannot be applied in biotyping of $Y$. enterocolitica.
\end{abstract}

Keywords: Yersinia enterocolitica, Pigs, Boars, Zoonotic strains, MALDI TOF MS, VITEK 2 Compact, PCR, Biotyping, 1B/O:8

\section{Background}

Yersinia enterocolitica, a causative agent of yersiniosis is widespread in an environment and animals [1]. Yersiniosis was the third most frequently reported zoonosis in the European Union in 2015, with the notification rate of 2.20 cases per 100,000 population which was $6.8 \%$ higher than in 2014 [2]. In 2014 in Poland 244 cases of

\footnotetext{
* Correspondence: gabriela.bugla-ploskonska@uwr.edu.pl

${ }^{1}$ Department of Microbiology, Faculty of Biological Sciences, Institute of Genetics and Microbiology, University of Wroclaw, S. Przybyszewskiego 63/ 77, 51-148 Wrocław, Poland

Full list of author information is available at the end of the article
}

yersiniosis were recorded $(0.63 / 100,000)$ [3]. Y. enterocolitica in humans may cause symptoms, i.e. fever, abdominal pain, diarrhea, vomiting, and pathologies such as self-limiting enteritis, acute mesenteric lymphadenitis and septicemia [4]. Pigs are generally considered a symptomless reservoir for this rod shaped bacterium. The wild boars are a new tested reservoir for Y. enterocolitica. The wild boar population in Europe and Poland has increased and venison consumption by humans is currently higher. Growing tendency of out-door pig farming is also noted [5-7]. Wild boars appear to be new, underestimated

(c) The Author(s). 2018 Open Access This article is distributed under the terms of the Creative Commons Attribution 4.0 International License (http://creativecommons.org/licenses/by/4.0/), which permits unrestricted use, distribution, and 
reservoir for $Y$. enterocolitica. Therefore, studies on the prevalence of $Y$. enterocolitica in wild boars can be considered important from the point of view of public health. Bancerz-Kisiel et al. in 2015 [8] isolated Y. enterocolitica from 26.5\% of 151 wild boars hunted during 2012/2013 season in central, northern and southern Poland and that was the first report on $Y$. enterocolitica wild boars prevalence in Poland. The research also undertaken by BancerzKisiel et al. [9] indicated the isolation of $Y$. enterocolitica from the half of 20 wild boar carcasses.

Despite numerous reports on the occurrence of $Y$. enterocolitica among humans, pigs or wild boars, the detection and identification are still difficult due to an overgrowth by bacteria from poly-contaminated samples, unusual growth characteristics of $Y$. enterocolitica and arduous discrimination of its colonies from other bacteria exhibiting Yersinia-like colonies on cefsulodin-irgasan-novobiocin Agar (CIN) such as Citrobacter freundii, C. braakii, Aeromonas hydrophila, Enterobacter cloacae, Providencia rettgeri, and Morganella morganii [10-12]. The recommended isolation of $Y$. enterocolitica is carried out by using ISO 10273-2003 method [13]. This method is recommended for both food and pig tonsils analyses. This protocol involves enrichment in peptone sorbitol bile (PSB) broth, and streaking on CIN. Another enrichment is also recommended, irgasan-ticarcillin-potassium chlorate (ITC) broth and streaking on Salmonella-Shigella-sodium-deoxycholate-calcium chloride (SSDC) agar plate [14]. However Fondrevez [15] modified this protocol and proved that streaking on CIN Agar from ITC broth could increase the number of positive samples in comparison with ISO 10273-2003 method [15]. The ITC/CIN culturing both favors the growth of biotype $4[16,17]$ which is the most common biotype in Europe within humans and also animals such as slaughtered pigs [18].

To isolate presumptive strains of $Y$. enterocolitica, the ITC broth and CIN agar media are recommended [3, 4]. CIN agar was devised by Schiemann in 1979 and is still useful in $Y$. enterocolitica isolation [10-12, 19-21]. Morphology of Yersinia sp. colony on CIN agar is known as red bull's eye, red centre with colourless translucent rim $[11,12]$.

Many methods have been designed for the identification of $Y$. enterocolitica [19]. In the present study we verified the Matrix-Assisted Laser Desorption Ionization-Time of Flight Mass Spectrometry (MALDI TOF MS) results using the VITEK 2 Compact system, and polymerase chain reaction (PCR) for identification of Y. enterocolitica isolated from humans and animal sources.

An alternative to time-consuming and biochemicalbased techniques is MALDI TOF MS, a suitable, rapid and powerful tool for identification of the $Y$. enterocolitica $[1,22-26]$. Some data confirmed its utility in the biotyping $[27,28]$. Biomarkers such as ribosomal, structural, and
DNA/RNA binding proteins produce the fingerprints that vary between different microorganisms and have spectrum specific to genus, species, and subspecies [29]. Despite the great advantages of MALDI TOF MS, there are also some limitations. The identification of environmental and potentially zoonotic strains is feasible only if the database of reference spectra contains fingerprints of genera, species or subspecies of required strains [30]. As yet MALDI TOF MS was applied mostly for clinical $Y$. enterocolitica strains [1, 24-27, 31]. Few authors report on application of MALDI TOF MS to identification of potentially zoonotic $Y$. enterocolitica strains [23, 28]. MALDI TOF MS was already used as a tool for identification of $Y$. enterocolitica strains isolated from slaughtered pigs and wild boars [32]. This work has not revealed the usefulness of MALDI TOF MS in zoonotic $Y$. enterocolitica strains identification but authors have confirmed all results using $16 \mathrm{~S}$ rDNA sequencing [32].

VITEK 2 Compact is automatic biochemical-based method which includes 48 biochemical features, being commonly used for microbial identification in clinical laboratories [33, 34]. VITEK 2 Compact allows microorganisms' identification up to $4 \mathrm{~h}$. Each well measures the metabolic activity of strain among ability to acidification, alkalization and enzymatic hydrolysis of substrates and bacterial growth in the presence of inhibitors. The system detects bacterial growth and metabolic changes in the microwells by using a fluorescence-based technology. Results of mentioned biotyping and biochemical-based methods may be influenced by conditions of bacterial incubation such as the composition of media or $\mathrm{pH}$ [34]. There is no available data on the utility of VITEK 2 Compact in identification of zoonotic $Y$. enterocolitica isolates.

A number of DNA-based methods for identification of zoonotic $Y$. enterocolitica strains such as PCR, and DNA sequencing was introduced [35]. Three of them have used the 16S rDNA-specific PCR designed by [36] for accurate and rapid species confirmation of $Y$. enterocolitica [36-38] In other PCR techniques identification of $Y$. enterocolitica were based on detection of particular species-specific virulence genes [10, 19, 32, 37, 38]. The $16 \mathrm{~S} \mathrm{rDNA}$ sequence analysis is still the gold standard in microbial identification. In this paper $16 \mathrm{~S}$ rDNA sequence analysis was used to reveal the species membership of three wild boars isolates.

Due to the biochemical characteristics and high heterogeneity $Y$. enterocolitica has been divided into the biotypes (Table 1) while the serotype is dependent on its lipopolysaccharide (LPS) structures. The biotype 1A is considered to be non-pathogenic to humans, whereas the biotype $1 \mathrm{~B}$, so-called American, is the most dangerous biotype within a species. The biotypes 2, 3, 4, 5 called European, are considered as weakly pathogenic. The most frequently used biotyping scheme was described in 1987 by Wauters [39]. 
Table 1 Biochemical tests used for Yersinia enterocolitica biotyping based on Wauters [39, 41]

\begin{tabular}{|c|c|c|c|c|c|c|c|c|c|}
\hline \multirow{2}{*}{\multicolumn{2}{|c|}{$\begin{array}{l}\text { Y. enterocolitica biotypes } \\
\text { pathogenicity against } \\
\text { humans }\end{array}$}} & \multicolumn{8}{|c|}{ Reaction of biotypes } \\
\hline & & Lipase $^{a}$ & Esculin & Indole & Xylose $^{a}$ & Trehalose $^{a}$ & Pyrazinamidase & $\beta$-D-Glucosidase ${ }^{a}$ & Voges-Proskauer \\
\hline Nonpathogenic & $1 \mathrm{~A}$ & + & + & + & + & + & + & + & + \\
\hline Highly pathogenic & $1 \mathrm{~B}$ & + & - & + & + & + & - & - & + \\
\hline \multirow[t]{4}{*}{ Weakly pathogenic } & 2 & - & - & $(+)$ & + & + & - & - & + \\
\hline & 3 & - & - & - & + & + & - & - & $+(\mathrm{c})$ \\
\hline & 4 & - & - & - & - & + & - & - & + \\
\hline & 5 & - & - & - & V & - & - & - & $(+)$ \\
\hline
\end{tabular}

() = Delayed reaction; $V$ = variable reaction; (c) Biotype of serotype O:3 found in Japan [39]; ${ }^{\text {a }}$ feature included in VITEK 2 Compact

Efficiency of biotyping may be limited by presence of unusual animals' strains. Within the biochemical features of VITEK 2 Compact only four of them are included in biotyping scheme proposed by Wauters. Since serotypes belonging to different biotypes $(1 \mathrm{~A}, 1 \mathrm{~B}, 2-5)$ may have a common O-specific LPS antigen, serotyping cannot be definite information on strain pathogenicity.

The main goal of present study was identification of presumptive $Y$. enterocolitica isolates using MALDI TOF MS. The consecutive aim was verification of MALDI TOF MS results of $Y$. enterocolitica using VITEK 2 Compact and PCR methods. Based on these results we investigated the incidence of $Y$. enterocolitica in humans and prevalence within pigs and wild boars during the years 2014-2015 in south-west Poland, where no research on this topic has been carried out yet.

\section{Methods}

\section{Bacterial isolates}

The bacterial isolates were obtained from 293 human faeces swabs routinely examined by $\mathrm{PhD}$ Kamila Korzekwa at Dialab Medical Laboratory (Wrocław, Poland). Bacteria were also isolated from 168 rectal and tonsillar swabs taken from healthy half-year old fattening pigs (Sus scrofa domestica), and from 130 swabs taken from tonsils and nasopharynx of hunted wild boars (Sus scrofa). Swabs from pigs and wild boars were taken by doctor of veterinary medicine Prof. Jarosław Bystron at two separate slaughterhouses and one meat processing plants in Wielkopolskie voivodeship (south-west Poland). All isolates isolated from separate individuals were deposited at the Collection of Department of Microbiology, Institute of Genetics and Microbiology, University of Wrocław (Table 3).

The $Y$. enterocolitica reference strains used as a control in this study were obtained from Polish Collection of Microorganisms (PCM, Wrocław, Poland): PCM 1879 (4/O:3), PCM 1880 (5/O:3), PCM 1881 (4/O:3), PCM 1882 (1B), PCM 1883 (1A/O:5), PCM 1884 (2/O:8) and from Department of Applied Microbiology,
Faculty of Biology, University of Warsaw, Poland: $Y$. enterocolitica 2/O:9.

\section{Isolation of $Y$. enterocolitica from environmental and clinical sources}

Swabs from human faeces, half-year pigs' tonsils or rectum and also wild boars tonsils and nasopharynx were incubated at $30{ }^{\circ} \mathrm{C}$ for $48 \mathrm{~h}$ in the ITC Broth (Biocorp, Warszawa, Poland) and next plated onto the CIN Agar (Biocorp, Warszawa, Poland) at $30{ }^{\circ} \mathrm{C}$ for $24 \mathrm{~h}$ [11]. The colonies obtained on the CIN Agar that represented the red bull's eye morphology [12] were taken for further analysis.

\section{MALDI-TOF MS analyses}

A bacterial suspension was made by mixing the couple of single colonies of actively growing cultures in $300 \mu \mathrm{L}$ of water which were fixed by the addition of $900 \mu \mathrm{L}$ absolute ethanol and followed by extraction procedure as previously described [40]. Bacterial strains were identified by MALDI-TOF MS and all analyses were performed with an UltrafleXtreme mass spectrometer (Bruker Daltonics, Germany) using the Biotyper 3.1 software and database containing 4613 entries. Identification by MALDI-TOF MS was twice repeated.

\section{Sample preparation and procedure of identification for VITEK 2 compact}

A sterile microloop was used to take a few colonies of a pure culture cultivated on Luria-Bertani Broth (Biocorp, Warszawa, Poland) for 18 to $24 \mathrm{~h}$. A bacterial suspension was adjusted to McFarland Turbidity Standard of 0.5-0.63 in $3 \mathrm{~mL}$ of a $0.45 \%$ sodium chloride solution with a VITEK 2 DensiChek (bioMérieux, Warszawa, Poland). The GN card was put on the cassette and placed in the instrument. The time interval between suspension preparation and card filling was less than $30 \mathrm{~min}$ to avoid changes in turbidity. Cards were incubated at $35.5 \pm 1{ }^{\circ} \mathrm{C}$. Each card was removed from the incubator every $15 \mathrm{~min}$ and automatically subjected for colorimetric readings. Results were read after $10 \mathrm{~h}-18 \mathrm{~h}$ of incubation. 


\section{Biotyping}

The biotyping of presumptive $Y$. enterocolitica isolates $n$ = 37 was carried out based on Wauters [39] with modifications [41]. Table 1 shows the scheme of reactions of the individual biotypes.

$\beta$-D-glucosidase activity was assayed using $0.1 \%$ 4-nitrophenyl- $\beta$-D-glucopyranoside (Sigma-Aldrich, St. Louis, MO, USA) as substrate in $0.0666 \mathrm{M}$ monosodium phosphate pH 6.0 (Avantor Performance Materials, Gliwice, Poland). The bacteria suspended in $250 \mu \mathrm{L}$ of physiologic saline to McFarland Turbidity Standard No. 3. were added to the equal volume of the substrate. Tubes were incubated at $30{ }^{\circ} \mathrm{C}$ for $24 \mathrm{~h}$. Appearance of yellow colour (assessed visually) caused by nitrophenol release was interpreted as positive reaction.

Pyrazinamidase test was performed on slants containing tryptic soy broth $30 \mathrm{~g} / \mathrm{L}$ (Biocorp, Warszawa, Poland), yeast extract $3 \mathrm{~g} / \mathrm{L}$ (Biocorp, Warszawa, Poland), pyrazinecarboxanamide $1 \mathrm{~g} / \mathrm{L}$ (Sigma-Aldrich, St. Louis, MO, USA), sodium chloride $5 \mathrm{~g} / \mathrm{L}$ (Chempur, Piekary Śląskie, Poland) and agar $20 \mathrm{~g} / \mathrm{L}$ (Biocorp, Warszawa, Poland) in $0.2 \mathrm{M}$ Tris-maleate Buffer ( $\mathrm{pH}$ 6.0). The cultures were grown on pyrazinamidase agar slants for $48 \mathrm{~h}$ at $30{ }^{\circ} \mathrm{C}$. Then $1 \mathrm{~mL}$ of freshly prepared $1.0 \%$ aqueous solution of ferrous ammonium sulphate (Avantor Performance Materials, Gliwice, Poland) was flooded over the slants. Development of pink colour (assessed visually) within $15 \mathrm{~min}$ indicated presence of pyrazinoic acid formed by bacterial pyrazinamidase.

Bromocresol purple broth was used to assess carbohydrate fermentation. Bromocresol purple broth included proteose peptone $10 \mathrm{~g} / \mathrm{L}$ (Biocorp, Warszawa, Poland), meat extract $1 \mathrm{~g} / \mathrm{L}$ (Biocorp, Warszawa, Poland), sodium chloride $5 \mathrm{~g} / \mathrm{L}$ (Chempur, Piekary Śląskie, Poland), bromocresol purple $0.02 \mathrm{~g} / \mathrm{L}$ (Avantor Performance Materials, Gliwice, Poland) as well as xylose (Sigma-Aldrich, St. Louis, MO, USA) and trehalose (Sigma-Aldrich, St. Louis, MO, USA) at final concentration of $1.0 \%$. Bacteria were cultured for $24 \mathrm{~h}$ at $37{ }^{\circ} \mathrm{C}$ in $4 \mathrm{~mL}$ of bromocresol purple broth. Appearance of yellow colour indicated ability of tested isolate to ferment trehalose or xylose.

Indole production was assessed using the tryptophan broth (Biocorp, Warszawa, Poland). Bacterial cultures were incubated for $24 \mathrm{~h}$ at $37^{\circ} \mathrm{C}$, each tube was then flooded by $0.2-0.3 \mathrm{~mL}$ Kovacs' reagent (Pro-Lab Diagnostics, Round Rock, TX, USA). Development of deep red colour on broth surface indicated ability of indol production by tested strain.

Voges-Proskauer test was assayed to confirm the acetoin production by $Y$. enterocolitica strains. Bacterial cultures were incubated for $48 \mathrm{~h}$ at $37{ }^{\circ} \mathrm{C}$ in Clark's medium containing glucose $5 \mathrm{~g} / \mathrm{L}$ (Avantor Performance Materials, Gliwice, Poland), dipotassium phosphate $5 \mathrm{~g} / \mathrm{L}$
(Chempur, Piekary Śląskie, Poland). After that $1 \mathrm{~mL}$ of 6.0\% $\alpha$-naphthol (Avantor Performance Materials, Gliwice, Poland) and $1 \mathrm{~mL}$ of $40 \%$ potassium hydroxide (Chempur, Piekary Śląskie, Poland) were added and shaken. The development of pink-to-ruby red colour in the medium indicated acetoin production by tested isolate.

Lipase production was checked on Agar medium with 1\% Tween 80 (Avantor Performance Materials, Gliwice, Poland). Development of opacity in the medium indicated lipase production by tested isolate.

Esculin hydrolysis was detected on Bile Esculin LABAgar (Biocorp, Warszawa, Poland). The esculetin, which is the product of esculin hydrolysis, reacts with the ferric citrate to form a dark brown or black colony, indicating ability of isolate to hydrolyse esculin.

\section{Serotyping}

After biotyping $Y$. enterocolitica isolates were serotyped using the commercial antisera polyvalent group O:1 O:2, O:3, O:5, O:8 and O:9 (Denka Seiken, Tokyo, Japan). Single colony of tested strain was suspended in physiological solution. A drop of antiserum was added to cell suspension and the agglutination process indicated a positive reaction between bacteria and antisera.

\section{DNA extraction}

Total bacterial DNA from overnight culture in Luria-Bertani Broth (Biocorp, Warszawa, Poland) was extracted using commercial Genomic Mini kit (A\&A Biotechnology, Gdynia, Poland) according to the manufacturer's protocol.

\section{Identification of $Y$. enterocolitica using PCR targeting $16 \mathrm{~S}$ rDNA gene}

The PCR method, using specific primers for $Y$. enterocolitica 16S rDNA, i.e., Y1 5'-AATACCGCATAACGTCTTC G-3' and Y2 5'-CTTCTTCTGCGAGTAACGTC-3' was applied to identify all $n=37$ isolates [36-38]. PCR was performed in a total volume of $25 \mu \mathrm{L}$ including $1,5 \mu \mathrm{L}$ of $25 \mathrm{mM} \mathrm{MgCl}{ }_{2}$ (Thermo Fisher Scientific, Waltham, MA, USA), $2.5 \mu \mathrm{L}$ of $10 \times$ buffer (Thermo Fisher Scientific, Waltham, MA, USA), $0.5 \mu \mathrm{L}$ of $10 \mathrm{mM}$ dNTP (Thermo Fisher Scientific, Waltham, MA, USA), $0.1 \mu \mathrm{L}$ of $100 \mu \mathrm{M}$ primers (Genomed, Warszawa, Poland), $0.2 \mu \mathrm{L}$ of Taq polymerase (Thermo Fisher Scientific, Waltham, MA, USA), and $1 \mu \mathrm{L}$ of template DNA. The amplification was carried out using a MJ Mini Thermal Cycler (Bio-Rad, Hercules, CA, USA) as follows: initial denaturation at $94{ }^{\circ} \mathrm{C}$ for $5 \mathrm{~min}$, followed by 30 cycles of denaturation $\left(94{ }^{\circ} \mathrm{C}, 30 \mathrm{~s}\right)$, annealing at $55{ }^{\circ} \mathrm{C}$ for $30 \mathrm{~s}$, and extension at $72{ }^{\circ} \mathrm{C}$ for $45 \mathrm{~s}$. DNA from reference $Y$. enterocolitica strains was used as positive control. DNA from reference Staphylococcus aureus strain was used as negative control. 


\section{S rDNA sequencing analysis}

The fragments of $16 \mathrm{~S}$ rDNA genes were amplified from bacterial DNA of strains $3 \mathrm{dz}, 4 \mathrm{dz}, 8 \mathrm{dz}$ and positive control $Y$. enterocolitica 2/O:9 using PCR with universal primers $16 S$ 27f- (5'-AGAGTTTGATCMTGGCTCAG-3) [42] and 16S 519r (5'-GwATTACCGCGGCkGCTG-3') [43] (Genomed, Warsaw, Poland), producing an amplicon of about $500 \mathrm{bp}$. PCRs were performed using $1 \mu \mathrm{L}$ of template, $2.5 \mu \mathrm{L}$ of $10 \times$ Dream Taq PCR buffer (Thermo Fisher Scientific, Waltham, MA, USA), $0.1 \mu \mathrm{L}$ of $100 \mu \mathrm{M}$ primers (Genomed, Warsaw, Poland), $0.5 \mu \mathrm{L}$ of $100 \mathrm{mM}$ dNTP (Thermo Fisher Scientific, Waltham, MA, USA), $0.5 \mathrm{U}$ of Dream Taq DNA polymerase (Thermo Fisher Scientific, Waltham, MA, USA), and sterile water added to a volume of $25 \mu \mathrm{L}$. Amplification was performed using a MJ Mini Thermal Cycler (Bio-Rad, Hercules, CA, USA). Thermocycling parameters were $94{ }^{\circ} \mathrm{C}$ for $2 \mathrm{~min}, 25$ cycles of $94{ }^{\circ} \mathrm{C}$ for $30 \mathrm{~s}, 50{ }^{\circ} \mathrm{C}$ for $30 \mathrm{~s}$ and $72{ }^{\circ} \mathrm{C}$ for $30 \mathrm{~s}$; and a final extension step at $72{ }^{\circ} \mathrm{C}$ for $5 \mathrm{~min}$. The products of PCR amplification were examined by gel electrophoresis, and stained with ethidium bromide. The amplicons were sequenced using Sanger capillary sequencing method (Genomed, Warsaw, Poland). The sequences were identified at species level using BLAST algorithm and NCBI GeneBank database. The following criteria were applied on sequence analysis: (i) isolate was identified at species level when its sequence had a similarity score of $\geq 99 \%$ with that of a reference GeneBank sequence, (ii) isolate was identified at genus level when the similarity score was $<99 \%$ and $\geq 95 \%$, and (iii) isolate was identified at family level when the similarity score was $<95 \%$.

\section{Electrophoretic analysis of PCR products}

The amplified products and GeneRuler DNA Ladder Mix (Thermo Fisher Scientific, Waltham, MA, USA) were resolved on 1.5\% agarose gel (Prona Agarose Basica Le GQT) containing $0.5 \mu \mathrm{g} / \mathrm{mL}$ ethidium bromide and documented using GelDocXR System (Bio-Rad, Hercules, CA, USA). PCRs on DNA from Y. enterocolitica yielded a PCR product of $330 \mathrm{bp}$.

\section{Data analysis}

In MALDI TOF MS bacterial identification was performed using Biotyper 3.0 software. Identification of isolates at species level with $\log$ scores $\geq 2.300$ were considered highly probable, log scores between 2.299 and 2.000 indicated high probability of identification at the genus level and probable identification at species level. Log scores between of 1.999 and 1.700 yielded probable identification at genus level. Scores $<1.700$ were interpreted as unreliable identification.

Confidence values of isolate identification in VITEK 2 Compact system were allocated to several categories, i.e., 96-99\% indicated excellent, 93-95\% very good and $89-92 \%$ good identification at the species level, whereas confidence value of $85-88 \%$ indicated acceptable or low identification at the genus level. Confidence values $<85 \%$ indicated unreliable identification.

The data of both methods were expressed at point system, wherein for the correct identification at the species or genus level awarded 3 and 1 point, respectively. For incorrect or low discrimination of identification was assigned 0 . The results of Yersinia spp. identification obtained from 3 systems (MALDI TOF MS, VITEK 2 Compact and $16 \mathrm{~S}$ rDNA PCR) were compared by using the chi square test for independent pairs with Yates' correction ( $n$ =37). The degree of concordance between MALDI TOF MS or VITEK ${ }^{\oplus} 2$ Compact and PCR method was analyzed by using the Spearman correlation. A $P$ value of $<0.05$ was considered statistically significant. Statistical analysis was performed using Statistica ver. 12 (StatSoft, Poland).

\section{Results \\ Isolation of bacteria on ITC/CIN}

The culture of analysed material on ITC and CIN media allowed to obtain 650 presumptive $Y$. enterocolitica colonies from 591 individuals (Table 3). All 650 isolates growing on CIN Agar as mannitol fermenting colonies and presenting the "red bull's eye" morphology were subjected to further MALDI TOF MS analysis.

\section{MALDI TOF MS identification and $Y$. enterocolitica occurrence}

MALDI TOF MS identified 37 isolates as $Y$. enterocolitica, including three human isolates, 28 pig' and six wild boar' isolates (Tables 2, 3). All pig and human isolates and two of six wild boar' isolates designated as $2 \mathrm{dz}, 5 \mathrm{dz}$ were identified using MALDI TOF MS with log scores ranging from 2.300 to 3.000 meaning highly probable species identification. The log score value of MALDI TOF MS for other wild boar' isolates designated as $3 \mathrm{dz}, 4 \mathrm{dz}$, $8 \mathrm{dz}, 10 \mathrm{dz}$ ranged from 2.000 to 2.299 indicated secure genus identification, and probable species identification.

Table 2 Yersinia sp. isolates $n=37$ used in this study and deposited at the Collection of Department of Microbiology, Institute of Genetics and Microbiology, University of Wrocław

\begin{tabular}{lll}
\hline Source of isolates & List of strains \\
\hline Dialab Medical Laboratory & Human faeces & 3d, 42d, 58d \\
Slaughterhouse & Slaughtered & $90 z, 119 z, 121 z, 163 z, 165 z$, \\
& pigs & $168 z, 169 z, 170 z, 171 z, 173 z$, \\
& $174 z, 175 z, 176 z, 177 z, 179 z$, \\
& $180 z, 182 z, 183 z, 184 z, 186 z$, \\
& $187 z, 188 z, 189 z, 190 z, 191 z$, \\
& $192 z, 193 z, 194 z$ \\
Meat processing plant & Wild boars & $2 d z, 3 d z, 4 d z, 5 d z, 8 d z, 10 d z$
\end{tabular}


Table 3 Yersinia sp. isolates isolation with ITC/CIN from wild boars, pigs and human's faeces in south-west Poland during 20142015, identified using MALDI TOF MS and bioserotyping

\begin{tabular}{|c|c|c|c|c|c|}
\hline \multirow{2}{*}{$\begin{array}{l}\text { Source of isolates } \\
\text { Type of swabs samples }\end{array}$} & \multicolumn{2}{|l|}{ Wild boars } & \multicolumn{2}{|c|}{ Slaughtered pigs } & \multirow{2}{*}{$\begin{array}{l}\text { Humans } \\
\text { Faeces }\end{array}$} \\
\hline & Tonsils & Nasopharynx & Rectum & Tonsils & \\
\hline Number of individuals & 10 & 120 & 36 & 132 & 293 \\
\hline Number of presumptive "red bull's eye" colonies on CIN Agar & 10 & 133 & 58 & 145 & 304 \\
\hline Number of positive Yersinia sp. samples / individuals (\%) & $6(6$ of 10$)$ & $0(0)$ & $25(69.4)$ & $3(2.3)$ & $3(1)$ \\
\hline \multirow[t]{3}{*}{ Bioserotype } & $1 \mathrm{~A} / \mathrm{O}: 9 n=1$ & - & 4/0:3 & 4/0:3 & $4 / 0: 3 n=2$ \\
\hline & $1 \mathrm{~A} / \mathrm{NT} n=2$ & & $n=25$ & $n=3$ & $1 \mathrm{~B} / \mathrm{O}: 8 n=1$ \\
\hline & NT/NT $n=3$ & & & & \\
\hline
\end{tabular}

$N T$ - nontypeable

Despite the recommendation of using ITC/CIN media for selective isolation of $Y$. enterocolitica growth and "red bull's eye" morphology of bacterial species other than $Y$. enterocolitica was observed. Other species identified using MALDI TOF MS included Rahnella aquatilis, Providencia rettgeri, Erwinia persicina, Serratia liquefaciens, Ewingella americana, Raoultella ornithinolytica. The most common non- $Y$. enterocolitica specimen was Aeromonas salmonicida ssp. salmonicida. Interestingly, all isolates isolated from wild boars, identified as $Y$. enterocolitica presented viscous and mucous morphology not observed in $Y$. enterocolitica isolates isolated from human stool or slaughtered pig tonsils.

Three of 293 human individuals were positive (1.0\%) for $Y$. enterocolitica. An isolate named, as $3 \mathrm{~d}$ and $42 \mathrm{~d}$ were isolated from stool of 5-year old boy and 2-year old boy, respectively. An isolate named, as $58 \mathrm{~d}(1 \mathrm{~B} / \mathrm{O}: 8)$ was isolated from stool of 69 -year old women. Their medical order was general examination of faeces. According to the MALDI TOF MS results six Yersinia sp. isolates were isolated from $n=10$ wild boars tonsils. In the wild boars' nasopharynx $(n=120)$ were not confirmed any of Yersinia sp. isolates. The number of pig' rectum swabs $(n=36)$ was the source for $25 Y$. enterocolitica isolates (69.4\% of number of rectum' swabs). The pig' tonsils swabs $(n=132)$ were the source for $3 Y$ Y enterocolitica isolates (2.3\% of number of tonsils' swabs) (Table 3$)$.

\section{VITEK $^{\circ} 2$ compact identification of $Y$. enterocolitica isolates}

All 37 isolates identified by MALDI TOF MS as Y. enterocolitica were then verified by VITEK 2 Compact. Results obtained from VITEK 2 Compact (all ranked excellent) agreed with MALDI TOF MS results for three human isolates, 28 pigs' isolates and four wild boar' isolates, i.e., $2 \mathrm{dz}$, $4 \mathrm{dz}, 5 \mathrm{dz}$ and $10 \mathrm{dz}(p=0.061)$.

However, two wild boar' isolates identified by MALDI TOF MS as $Y$. enterocolitica, i.e., $3 \mathrm{dz}$ and $8 \mathrm{dz}$ were identified by VITEK $^{\bullet} 2$ Compact as $Y$. kristensenii (Table 4) with excellent and very good confidence, respectively.

\section{PCR-identification of $Y$, enterocolitica isolates}

PCRs targeting $Y$. enterocolitica-specific $16 \mathrm{~S}$ rDNA produced a 330-bp product for 34 isolates identified previously as $Y$. enterocolitica using both, MALDI TOF MS $(P=0.31)$ and VITEK $^{\circ} 2$ Compact $(P=0.61)$. However, three isolates isolated form wild boars, i.e., $3 \mathrm{dz}, 4 \mathrm{dz}, 8 \mathrm{dz}$, and identified using both, MALDI TOF MS and VITEK 2 Compact have not produced a 330-bp product in PCR. $16 \mathrm{~S}$ rDNA sequencing analysis of these three strains (Table 4) revealed with a similarity score of $\geq 99 \%$ with a reference GeneBank sequence that $3 \mathrm{dz}$ and $4 \mathrm{dz}$ are $Y$. kristensenii, and $8 \mathrm{dz}$ is Y. pekkanenii.

\section{Bioserotyping of $Y$. enterocolitica isolates}

Identification by VITEK 2 Compact based on 48 biochemical features gave results for four reactions in the biotyping scheme. However biotyping was also performed on 37 isolates according to Wauters scheme [39]. Results of incubation using Wauters methodology and results of VITEK 2 Compact differed in xylose fermentation for all wild boar' isolates and one human isolate. VITEK 2 Compact did not detect lipase production however detected the $\beta$-D-glucosidase production of $8 \mathrm{dz}$ wild boar' isolates. The differences in reactions were listed in Table 5.

All pig' $Y$. enterocolitica isolates together with two of three human' isolates were assigned to the biotype 4. All these isolates were identified as O3 serogroup. Remaining human $Y$. enterocolitica isolate was assigned to bioserotype 1B/O:8. Only $1 Y$. enterocolitica isolate from wild boar' tonsils $(2 \mathrm{dz})$ could be bioserotyped as 1A/O:9. Two wild boar' isolates $(5 \mathrm{dz}, 10 \mathrm{dz})$ were assigned to $1 \mathrm{~A}$ biotype but were nontypeable using antisera, while three other wild boar' isolates ( $3 \mathrm{dz}, 4 \mathrm{dz}, 8 \mathrm{dz}$ ) could not be bioserotyped (Table 3).

\section{Discussion}

In recent years incidence of yersiniosis in Poland has remained at a stable level, but data about number of cases can be affected by low reportability, lack of bioserotyping as a part of routine diagnostics and underreporting of extraintestinal yersiniosis. In Poland prevalence of 
Table 4 The differences between results obtained by independent methods: MALDI TOF MS, VITEK ${ }^{\circledR} 2$ Compact, PCR with specific for Y. enterocolitica 165 rDNA gene and 165 rDNA sequencing. Wild boar' Yersinia sp. isolates $n=3$

\begin{tabular}{|c|c|c|c|c|c|}
\hline Strain no. & Bioserotype & $\begin{array}{l}\text { MALDI TOF } \\
\text { Biotyper }\end{array}$ & $\begin{array}{l}\text { VITEK } 2 \text { Compact } \\
\text { bioMérieux }\end{array}$ & $\begin{array}{l}\text { PCR targeting } 16 S \\
\text { rDNA gene of } Y \text {. } \\
\text { enterocolitica }\end{array}$ & $\begin{array}{l}16 \mathrm{~S} \text { rDNA } \\
\text { Sequencing } \\
\text { Analysis }\end{array}$ \\
\hline $3 d z$ & NT & $\begin{array}{l}\text { Yersinia } \\
\text { enterocolitica }\end{array}$ & Yersinia kristensenii & - & Yersinia kristensenii \\
\hline $4 d z$ & NT & $\begin{array}{l}\text { Yersinia } \\
\text { enterocolitica }\end{array}$ & $\begin{array}{l}\text { Yersinia } \\
\text { enterocolitica }\end{array}$ & - & Yersinia kristensenii \\
\hline $8 d z$ & NT & $\begin{array}{l}\text { Yersinia } \\
\text { enterocolitica }\end{array}$ & Yersinia kristensenii & - & Yersinia pekkanenii \\
\hline
\end{tabular}

NT: nontypeable

- lack of amplification product

$Y$. enterocolitica in animals was studied in the north-east region of the country only $[8,19,44,45]$.

$Y$. enterocolitica has been isolated all over the world not only from pigs, but also from wild boars, sheep, horses, cattle, dogs, cats, ducks, amphibians and even beavers [10, 44-48]. The research of Bancerz-Kisiel [8] indicated the presence of $Y$. enterocolitica in 26, 5\% of 151 wild boars, thus game animals were considered a potential reservoir of this pathogen. The role of wild boar, belonging together with pig to the same species (Sus scrofa), in Y. enterocolitica carriage is poorly understood [49]. In contrast, the presence of $Y$. enterocolitica in pigs is observed all over the world, accounting for increasing problem in food hygiene. We revealed that wild boars could be considered a source of $Y$. enterocolitica. However, due to some specific characteristics identification of $Y$. enterocolitica isolates from wild boars needs more effort than that from other sources.

There are many concerns on the usefulness of recommended methods for isolation and identification of $Y$. enterocolitica [5, 12, 50,51]. Due to the overgrowth by surrounding microflora selective enrichment and culturing are needed. Isolation method using ITC/CIN media is recommended, but their sensitivity and discriminatory power are lower compared to other methods. This method takes at least $72 \mathrm{~h}$ to obtain presumptive $Y$. enterocolitica colonies on CIN agar. In turn, this method is much faster as compared to cold enrichment which usually includes 3 -weeks incubation at $4{ }^{\circ} \mathrm{C}$. Isolation of $Y$. enterocolitca using ITC/CIN has some limitations because many other species such as certain mannitol-fermenting bacteria can efficiently grow on these media. Moreover it is well known that ITC/CIN culturing favours the growth of biotype 4 . In this work we report successful isolation of $Y$. enterocolitca bioserotype $1 \mathrm{~B} /$ $\mathrm{O}: 8$ from ITC/CIN. The identification of isolates may be difficult due to variability of bacterial strains in terms of biochemical characteristics [5]. In this work the MALDI TOF MS was used in order to identify presumptive $Y$. enterocolitica isolates. To confirm identification results of potentially zoonotic isolates we used VITEK $^{\circ} 2 \mathrm{Com}^{-}$ pact, bioserotyping, and PCR.

MALDI TOF MS has been shown to be a common method in $Y$. enterocolitica identification [1, 26-28]. However, the use of both, MALDI TOF MS as well as VITEK ${ }^{\circ}$ 2 Compact may have limitations since resources they rely on are dominated by the data from clinical isolates. Some authors reported the consistence of MALDI TOF MS results with Vitek 2 for $Y$. enteroclitica identification, but most of this research was also conducted on clinical isolates [25]. MALDI TOF MS and VITEK 2 Compact can be thus considered first-line method for identification of $Y$. enterocolitica isolates in clinical microbiology but its use is still developing in identification of zoonotic isolates.

Table 5 The differences in biochemical reaction results of selected Yersinia sp. isolates according to the Wauters scheme [39] and VITEK ${ }^{\circledR}$ 2 Compact

\begin{tabular}{|c|c|c|c|c|c|c|c|c|}
\hline \multirow[t]{2}{*}{ Strain no. } & \multicolumn{4}{|c|}{ Reaction of biotypes based on Wauters methodology } & \multicolumn{4}{|c|}{ Reaction of biotypes based on VITEK 2 Compact results } \\
\hline & Lipase & Xylose & Trehalose & $\beta$-D-glucosidase & Lipase & Xylose & Trehalose & $\beta$-D-glucosidase \\
\hline $58 d$ & + & + & + & - & - & - & + & - \\
\hline $2 d z$ & + & + & + & + & - & - & + & + \\
\hline $3 d z$ & - & + & + & - & - & - & + & - \\
\hline $4 d z$ & - & + & + & - & - & - & + & - \\
\hline $5 \mathrm{dz}$ & + & + & + & + & - & - & + & + \\
\hline $8 d z$ & - & + & + & - & - & - & + & + \\
\hline $10 d z$ & + & + & + & + & - & - & + & + \\
\hline
\end{tabular}


Zoonotic $Y$. enterocolitica isolates used in this study are characterized by low diversity. Each method applied by us gave reliable and repeatable results, however they produced different results from each other especially for wild boar' isolates. We thus included a PCR-based method, targeting $16 \mathrm{~S}$ rDNA gene, and compared to the results by MALDI TOF MS and VITEK 2 Compact. For most $Y$. enterocolitica isolates we demonstrated high agreement of identification results using 3 above methods. However, 3 isolates from wild boars identified as $Y$. enterocolitica by MALDI TOF MS, could not produce species-specific PCR amplicon for $16 \mathrm{~S}$ rDNA gene. Based on $16 \mathrm{~S}$ rDNA sequencing analysis these isolates were assigned to species other than $Y$. enterocolitca. These results indicate the necessity of using multiple approaches for identification of $Y$. enterocolitica from wild boars. These animals are generally considered an important reservoir of pathogenic Yersinia species [7] but in this work only nonpathogenic $Y$. enterocolitica isolates were isolated from wild boars, as inferred from biotyping results. The recent report of Magistrali et al. [52] has revealed that wild boars could act as a reservoir of atypical $Y$. pseudotuberculosis, other enteropathogenic species within the Yersinia genus, which were associated with severe symptoms and death of deer and macaques. As mentioned above, other probable cause of isolate misidentification can be overrepresentation of clinical strains in databases. Although these methods are often used in environmental microbiology and databases are successively expanding. Fredriksson-Ahoma et al. [6] has reported some data about the differences between $Y$. enterocolitica isolated from wild boars and domestic pigs. The differences were related to distribution of bioserotypes, virulence genes content, genotype and sensitivity to antimicrobials. In this paper [6] it was also suggested that more wild boar' isolates have to be characterized to verify similarity between $Y$. enterocolitca from wild boars and pigs. Based on mentioned reports $[6,7,52]$ we suppose that wild boars can harbor specific Yersinia population which may pose difficulties to standard identification.

In this work we have proven that application of single method is not sufficient for reliable identification of $Y$. enterocolitica. The discrepancy in species identification noted for certain wild boar isolates may indicate that Yersinia sp. and $Y$. enterocolitica identifiaction may need further verification. The need for application of multiple methods was already noted for the E. coli identification [34].

The Wauters' scheme reactions were conducted individually. We did not considered results obtained from VITEK $^{\bullet} 2$ Compact to biotyping of our isolates, because its results were incompatible with Wauters' scheme (Table 5). The biotyping of pig' isolates proved the presence of $Y$. enterocolitica strains pathogenic for humans, which is consistent with the European reports [2].
Our data revealed that prevalence of $Y$. enterocolitica was higher in rectal swabs as compared to the tonsillar swabs (Table 3). It could be hypothesized that pigs examined here were in the early infection stage, when bacteria were only locally disseminated. The late infection stage is characterized by $Y$. enterocolitica presence within the lymphoid tissue e.g. tonsils, when bacteria spread through the organism [53]. In pigs slaughtered at the age of 135 days or more, the tonsils may be a more significant source of $Y$. enterocolitica than faeces $[54,55]$. Pigs tested in this work were at the age of 180 days, which does not support the above hypothesis. To characterize the carrier state in pigs it is necessary to confirm if during the sampling pigs were infected systemically or locally. In addition the younger pigs should be examined for Y. enterocolitica occurrence.

\section{Conclusion}

MALDI TOF MS can be successfully used for Yersinia sp. identifiaction from human' and pig' samples, but identification of wild boar' isolates needs additional tests. The biochemical tests included in VITEK $^{\circ} 2$ Compact are not suitable for biotyping of $Y$. enterocolitica. Yersinia sp. isolates from wild boar appear to be more heterogenous than isolates from previously characterized sources i.e. humans and pigs. Thus, the results of the present study strengthen the necessity of use of multiple methods for identification of zoonotic $Y$. enterocolitica. We found that pathogenic $1 \mathrm{~B}$ and 4/O:3 isolates of $Y$. enterocolitica are present in south-west Poland within humans and pigs.

\section{Abbreviations}

CIN: Cefsulodin-irgasan-novobiocin; ITC: Irgasan-ticarcillin-potassium chlorate; LPS: Lipopolysaccharide; MALDI TOF MS: Matrix Assisted Laser Desorption Ionization Time of Flight Mass Spectrometry; PCR: Polymerase chain reaction; SSDC: Salmonella-Shigella-sodium-deoxycholate-calcium chloride

\section{Acknowledgements}

We thank the Dialab, Medical Laboratory, Wrocław, Poland for supplying all clinical isolates and contribution analysis tool VITEK 2 Compact. We thank Professor Andrzej Gamian for collaboration possibility with Polish Collection of Microorganisms. We also thank Anna Tobiasz and Klaudia Rogala from Polish Collection of Microorganisms for excellent technical support. We thank Kate and Gene Scheck- English speaking people who corrected the paper. We thank to PhD Marta Nieckarz and Professor Katarzyna Brzostek for supplying reference strains of $Y$. enterocolitica.

\section{Funding}

The publication was supported by the Wroclaw Centre of Biotechnology, programme The Leading National Research Centre (KNOW) for years 20142018. This work was supported in part by special-purpose grant of the Ministry of Science and Higher Education to carry out research or development work and tasks related to the development of young scientists and doctoral participants [Faculty of Biological Sciences, University of Wroclaw, 0420/ 1404/16] (Main Investigator: Katarzyna Morka, Principal Investigator of the Team: Gabriela Bugla-Płoskońska). The funders had no role in study design, data collection and analysis, interpretation of data, nor writing the manuscript, decision to publish, or preparation of the manuscript.

\section{Availability of data and materials}

All data generated or analysed during this study are available from the corresponding author on reasonable request. 


\section{Authors' contributions}

$\mathrm{KM}, \mathrm{GBP}$, JB wrote the paper. KM, GBP, JB, AKK participated in discussion of the study. KM, GBP, JB coordinated the study and drafted the manuscript. KM participated in all part of experiments. JBy supplied all animals' swabs. AKK contributed reagents/materials/analysis tools. KK coordinated isolation and identification of humans' isolates using VITEK 2 Compact. KGR did the statistical analyses. All the authors read and approved the final manuscript.

\section{Ethics approval and consent to participate}

Not applicable.

\section{Consent for publication}

Not applicable.

\section{Competing interests}

The authors declare that they have no competing interests.

\section{Publisher's Note}

Springer Nature remains neutral with regard to jurisdictional claims in published maps and institutional affiliations.

\section{Author details}

'Department of Microbiology, Faculty of Biological Sciences, Institute of Genetics and Microbiology, University of Wroclaw, S. Przybyszewskiego 63/ 77, 51-148 Wrocław, Poland. ${ }^{2}$ Faculty of Veterinary Medicine, Department of Food Hygiene and Consumer Health Protection, Wroclaw University of Environmental and Life Sciences, Norwida 31, 50-375 Wrocław, Poland. ${ }^{3}$ Polish Collection of Microorganisms, Ludwik Hirszfeld Institute of Immunology and Experimental Therapy, Polish Academy of Sciences, $\mathrm{R}$. Weigla 12, 53-114 Wrocław, Poland.

\section{Received: 15 March 2017 Accepted: 6 August 2018}

\section{Published online: 17 August 2018}

\section{References}

1. Zacharczuk K, Sulikowska A, Nowacka E, Szych J, Gierczyński R. Rapid identification of Yersinia enterocolitica by matrix-assisted laser desorption/ ionization time-of-flight mass spectrometry (MALDI-TOF MS). Med Dosw Mikrobiol. 2012;64:123-8

2. European Food Safety Authority, European Centre for Disease Prevention and Control. The European Union summary report on trends and sources of zoonoses, zoonotic agents and food-borne outbreaks in 2015. EFSA J. 2016; http://doi.wiley.com/10.2903/j.efsa.2016.4634. Accessed 22 Feb 2017.

3. Kamińska S, Sadkowska-Todys M. Yersiniosis in Poland in 2014. Przegl Epidemiol. 2016:70:367-74.

4. Skorek K, Raczkowska A, Dudek B, Miętka K, Guz-Regner K, Pawlak A, Klausa E, Bugla-Płoskońska G, Brzostek K. Regulatory protein OmpR influences the serum resistance of Yersinia enterocolitica 0:9 by modifying the structure of the outer membrane. PloS One. 2013:8(11):e79525.

5. Fredriksson-Ahomaa M, Korkeala H. Low occurrence of pathogenic Yersinia enterocolitica in clinical, food, and environmental samples: a methodological problem. Clin Microbiol Rev. 2003:16:220-9.

6. Fredriksson-Ahomaa M, Wacheck S, Bonke R, Stephan R. Different enteropathogenic Yersinia strains found in wild boars and domestic pigs. Foodborne Pathog Dis. 2011;8:733-7.

7. Fredriksson-Ahomaa M, Wacheck S, Koenig M, Stolle A, Stephan R. Prevalence of pathogenic Yersinia enterocolitica and Yersinia pseudotuberculosis in wild boars in Switzerland. Int J Food Microbiol. 2009; 15(135):199-202

8. Bancerz-Kisiel A, Platt-Samoraj A, Szczerba-Turek A, SyczyłO K, Szweda W. The first pathogenic Yersinia enterocolitica bioserotype 4/0:3 strain isolated from a hunted wild boar (Sus scrofa) in Poland. Epidemiol Infect. 2015;143: 2758-65.

9. Bancerz-Kisiel A, Socha P, Szweda W. Detection and characterisation of Yersinia enterocolitica strains in cold-stored carcasses of large game animals in Poland. Vet J Lond Engl. 2016;208:102-3.

10. Platt-Samoraj A, Ugorski M, Szweda W, Szczerba-Turek A, Wojciech K, Procajło Z. Analysis of the presence of ail, ystA and ystB genes in Yersinia enterocolitica strains isolated from aborting sows and aborted fetuses. J Vet Med B Infect Dis Vet Public Health. 2006;53:341-6.
11. Savin C, Leclercq A, Carniel E. Evaluation of a single procedure allowing the isolation of enteropathogenic Yersinia along with other bacterial enteropathogens from human stools. PLoS One. 2012;7(7):e41176.

12. Tan LK, Ooi PT, Carniel E, Thong KL. Evaluation of a modified Cefsulodin-IrgasanNovobiocin Agar for isolation of Yersinia spp. PloS One. 2014;9(8):e106329.

13. ISO 10273:2003. Microbiology of food and animal feeding stuffs - Horizontal method for the detection of presumptive pathogenic Yersinia enterocolitica: ISO. https://www.iso.org/standard/34564.html. Accessed 22 Feb 2017.

14. Denis M, Houard E, Labbé A, Fondrevez M, Salvat G. A Selective chromogenic plate, YECA, for the detection of pathogenic Yersinia enterocolitica: Specificity, sensitivity, and capacity to detect pathogenic $Y$. enterocolitica from pig tonsils. J Pathog. 2011;2011:296275.

15. Fondrevez M, Labbé A, Houard E, Fravalo P, Madec F, Denis M. A simplified method for detecting pathogenic Yersinia enterocolitica in slaughtered pig tonsils. J Microbiol Methods. 2010:83:244-9.

16. De Zutter L, Le Mort L, Janssens M, Wauters G. Short-comings of irgasan ticarcillin chlorate broth for the enrichment of Yersinia enterocolitica biotype 2, serotype 9 from meat. Int J Food Microbiol. 1994;23:231-7.

17. Schiemann DA. Development of a two-step enrichment procedure for recovery of Yersinia enterocolitica from food. Appl Environ Microbiol. 1982;43:14-27.

18. Schneeberger M, Brodard I, Overesch G. Virulence-associated gene pattern of porcine and human Yersinia enterocolitica biotype 4 isolates. Int J Food Microbiol. 2015;198:70-4.

19. Syczyło K, Platt-Samoraj A, Bancerz-Kisiel A, Szczerba-Turek A, Lipczyńska K Jabłoński A, Procajło Z, Szweda W. Monitoring of Yersinia enterocolitica strains from free-living animals using different methods. Pol J Vet Sci. 2016;19:221-3.

20. Bottone EJ. Yersinia enterocolitica: the charisma continues. Clin Microbiol Rev. 1997:10:257-76.

21. Bottone EJ. Yersinia enterocolitica: overview and epidemiologic correlates. Microbes Infect Inst Pasteur. 1999:1:323-33.

22. Bardoň J, Štromerová N. Identification of zoonotic bacterial pathogens by the MALDI TOF MS method. Klin Mikrobiol Infekční Lékařství. 2015:21:46-50.

23. Stamm I, Hailer M, Depner B, Kopp PA, Rau J. Yersinia enterocolitica in diagnostic fecal samples from European dogs and cats: identification by fourier transform infrared spectroscopy and matrix-assisted laser desorption ionization-time of flight mass spectrometry. J Clin Microbiol. 2013:51: 887-93.

24. Ayyadurai S, Flaudrops C, Raoult D, Drancourt M. Rapid identification and typing of Yersinia pestis and other Yersinia species by matrix-assisted laser desorption/ionization time-of-flight (MALDI-TOF) mass spectrometry. BMC Microbiol. 2010;10:285

25. Lasch P, Drevinek M, Nattermann H, Grunow R, Stämmler M, Dieckmann R, Schwecke T, Naumann D. Characterization of Yersinia using MALDI-TOF mass spectrometry and chemometrics. Anal Chem. 2010;82:8464-75.

26. Deng J, Fu L, Wang R, Yu N, Ding X, Jiang L, Lin L, Wang Y, Che X Comparison of MALDI-TOF MS, gene sequencing and the Vitek 2 for identification of seventy-three clinical isolates of enteropathogens. J Thorac Dis. 2014;6:539-44

27. Rizzardi K, Wahab T, Jernberg C. Rapid subtyping of Yersinia enterocolitica by matrix-assisted laser desorption ionization-time of flight mass spectrometry (MALDI-TOF MS) for diagnostics and surveillance. J Clin Microbiol. 2013;51: 4200-3

28. Stephan R, Cernela N, Ziegler D, Pflüger V, Tonolla M, Ravasi D, FredrikssonAhomaa M, Hächler $\mathrm{H}$. Rapid species specific identification and subtyping of Yersinia enterocolitica by MALDI-TOF mass spectrometry. J Microbiol Methods. 2011:87:150-3.

29. De Carolis E, Vella A, Vaccaro L, Torelli R, Spanu T, Fiori B, Posteraro B, Sanguinetti M. Application of MALDI-TOF mass spectrometry in clinical diagnostic microbiology. J Infect Dev Ctries. 2014;8:1081-8.

30. Singhal N, Kumar M, Kanaujia PK, Virdi JS. MALDI-TOF mass spectrometry: an emerging technology for microbial identification and diagnosis. Front Microbiol. 2015:6:791.

31. Clark AE, Kaleta EJ, Arora A, Wolk DM. Matrix-assisted laser desorption ionization-time of flight mass spectrometry: a fundamental shift in the routine practice of clinical microbiology. Clin Microbiol Rev. 2013;26: 547-603.

32. von Altrock A, Seinige D, Kehrenberg C. Yersinia enterocolitica isolates from wild boars hunted in Lower Saxony, Germany. Appl Environ Microbiol. 2015; 81(14):4835-40.

33. bioMérieux Clinical Diagnostics. Available from: http://www.biomerieuxdiagnostics.com/. Accessed 13 May 2016. 
34. Książczyk M, Kuczkowski M, Dudek B, Korzekwa K, Tobiasz A, Korzeniowska-Kowal A, Paluch E, Wieliczko A, Bugla-Płoskońska G. Application of routine diagnostic procedure, VITEK 2 compact, MALDI-TOF MS, and PCR assays in identification procedure of bacterial strain with ambiguous phenotype. Curr Microbiol. 2016;72: 570-82.

35. Bhaduri S, Cottrell B, Pickard AR. Use of a single procedure for selective enrichment, isolation, and identification of plasmid-bearing virulent Yersinia enterocolitica of various serotypes from pork samples. Appl Environ Microbiol. 1997;63:1657-60.

36. Neubauer $\mathrm{H}$, Hensel A, Aleksic S, Meyer H. Identification of Yersinia enterocolitica within the genus Yersinia. Syst Appl Microbiol. 2000;23:58-62.

37. Momtaz H, Davood Rahimian M, Safarpoor DF. Identification and characterization of Yersinia enterocolitica isolated from raw chicken meat based on molecular and biological techniques. J Appl Poult Res. 2013;22: $137-45$.

38. Wannet WJB, Reessink M, Brunings HA, Maas HME. Detection of pathogenic Yersinia enterocolitica by a rapid and sensitive duplex PCR assay. J Clin Microbiol. 2001;39:4483-6.

39. Wauters G, Kandolo K, Janssens M. Revised biogrouping scheme of Yersinia enterocolitica. Contrib Microbiol Immunol. 1987;9:14-21.

40. Król J, Wanecka A, Twardoń J, Mrowiec J, Dropińska A, Bania J, Podkowik M, Korzeniowska-Kowal A, Paściak M. Isolation of Staphylococcus microti from milk of dairy cows with mastitis. Vet Microbiol. 2016;182:163-9.

41. Nutrition C for FS and A. Laboratory Methods - BAM: Yersinia enterocolitica. Available from: http://www.fda.gov/Food/FoodScienceResearch/ LaboratoryMethods/ucm072633.htm. Accessed 14 Apr 2016.

42. Lane DJ. 16S/23S rRNA sequencing. In: Stackebrandt E, Goodfellow M, editors. Nucleic acid techniques in bacterial systematics. New York: Wiley; 1991. p. 115-75.

43. Turner S, Pryer KM, Miao VP, Palmer JD. Investigating deep phylogenetic relationships among cyanobacteria and plastids by small subunit rRNA sequence analysis. J Eukaryot Microbiol. 1999:46:327-38.

44. Bancerz-Kisiel A, Szweda W. Yersiniosis - a zoonotic foodborne disease of relevance to public health. Ann Agric Environ Med AAEM. 2015;22:397-402.

45. Bancerz-Kisiel A, Szczerba-Turek A, Platt-Samoraj A, Socha P, Szweda W. Bioserotypes and virulence markers of $Y$. enterocolitica strains isolated from roe deer (Capreolus capreolus) and red deer (Cervus elaphus). Pol J Vet Sci. 2014;17:315-9

46. Jagielski M, Rastawicki W, Kałuzewski S, Gierczyński R. Yersiniosisunappreciated infectious disease. Przegl Epidemiol. 2002;56:57-64.

47. Botzler RG, Wetzler TF, Cowan AB. Yersinia enterocolitica and Yersinia-like organisms isolated from frogs and snails. Wildl Dis. 1968;4:110-5.

48. Kot B, Piechota M, Jakubczak A. Analysis of occurrence of virulence genes among Yersinia enterocolitica isolates belonging to different biotypes and serotypes. Pol J Vet Sci. 2010;13:13-9.

49. Szweda W, Siemionek J, Procajło Z, Bancerz-Kisiel A, Platt-Samoraj A Szczerba-Turek A. Wild boars - the important reservoir of pathogenic microorganisms for pigs. Magazyn Weterynaryjny, Choroby Świń Monografia. 2009;1:601-4. [Article in Polish].

50. Knutsson R, Blixt $Y$, Grage $H$, Borch E, Rådström P. Evaluation of selective enrichment PCR procedures for Yersinia enterocolitica. Int J Food Microbiol. 2002;73:35-46

51. Van Damme I, Berkvens D, Botteldoorn N, Dierick K, Wits J, Pochet B, De Zutter L. Evaluation of the ISO 10273:2003 method for the isolation of human pathogenic Yersinia enterocolitica from pig carcasses and minced meat. Food Microbiol. 2013;36:170-5.

52. Magistrali CF, Cucco L, Manuali E, Sebastiani C, Farneti S, Ercoli L, Pezzotti G. Atypical Yersinia pseudotuberculosis serotype 0:3 isolated from hunted wild boars in Italy. Vet Microbiol. 2014;171:227-31.

53. Sansonetti PJ. War and peace at mucosal surfaces. Nat Rev Immunol. 2004;4: 953-64.

54. Nesbakken T, Iversen T, Eckner K, Lium B. Testing of pathogenic Yersinia enterocolitica in pig herds based on the natural dynamic of infection. Int J Food Microbiol. 2006;111:99-104.

55. Gürtler M, Alter T, Kasimir S, Linnebur M, Fehlhaber K. Prevalence of Yersinia enterocolitica in fattening pigs. J Food Prot. 2005;68:850-4.

Ready to submit your research? Choose BMC and benefit from:

- fast, convenient online submission

- thorough peer review by experienced researchers in your field

- rapid publication on acceptance

- support for research data, including large and complex data types

- gold Open Access which fosters wider collaboration and increased citations

- maximum visibility for your research: over $100 \mathrm{M}$ website views per year

At BMC, research is always in progress.

Learn more biomedcentral.com/submissions 